\title{
Effects of cerebrolysin on functional outcome of patients with traumatic brain injury: a systematic review and meta-analysis
}

This article was published in the following Dove Medical Press journal: Neuropsychiatric Disease and Treatment

\author{
Fariborz Ghaffarpasand' \\ Saeed Torabi² \\ Ali Rasti ${ }^{3}$ \\ Mohammad Hadi Niakan ${ }^{4}$ \\ Sara Aghabaklou 5 \\ Fatemeh Pakzad ${ }^{6}$ \\ Maryam Sadat Beheshtian ${ }^{7}$ \\ Reza Tabrizi ${ }^{8}$
}

'Research Center for Neuromodulation and Pain, Shiraz University of Medical Sciences, Shiraz, Iran; ${ }^{2}$ Department of Anesthesiology and Intensive Care Medicine, University Hospital of Cologne, Cologne, Germany; ${ }^{3}$ Poostchi Ophthalmology Research Center, Shiraz University of Medical Sciences, Shiraz, Iran; ${ }^{4}$ Trauma Research Center, Rajaei Trauma Hospital, Shiraz University of Medical Sciences, Shiraz, Iran; ${ }^{5}$ Department of Neurosurgery, Tehran University of Medical Sciences, Tehran, Iran; ${ }^{6}$ Department of Anesthesiology, Shiraz University of Medical Sciences, Shiraz, Iran; ${ }^{7}$ Department of Neurosurgery, Shahid Beheshti University of Medical Sciences, Tehran, Iran; ${ }^{8} \mathrm{Health}$ Policy Research Center, Institute of Health, Student Research Committee, Shiraz University of Medical Sciences, Shiraz, Iran

Correspondence: Mohammad Hadi Niakan

Trauma Research Center, Rajaei Trauma Hospital, Shiraz University of Medical Sciences, 6th Floor, Chamran Avenue, Shiraz 48I8684668, Iran

Tel +9892I 6707983

Email hadiniakan@yahoo.com
Background: Traumatic brain injury (TBI) remains a main public health problem being associated with high mortality and morbidity. The functional outcome of TBI remains unfavorable despite several surgical and medical therapies. Cerebrolysin is a neuropeptide with potential neuroregenerative entities.

Objective: The aim of the current systematic review and meta-analysis was to investigate the effects of cerebrolysin on functional outcome in patients with moderate and severe TBI.

Data sources: Online databases used included Medline, Scopus, EMBASE, Google Scholar, Web of Science, and Cochrane Library.

Study eligibility criteria: All the relevant studies with randomized clinical trial and cohort design evaluating the effects of intravenous cerebrolysin vs placebo on functional outcome of patients with TBI within the English literature up to October 2018 were included.

Study appraisal and synthesis methods: The articles were reviewed by two independent authors and the data were extracted to a data sheet. $I^{2}$ and Cochran's $Q$-statistics were used to assess heterogeneity. Based on the presence of significant heterogeneity across included studies, data were pooled using random-effects model with Dersimonian-Laird method and presented as standardized mean differences (SMDs) and corresponding 95\% CI.

Results: Five articles (5,685 participants) were included in the current meta-analysis. The overall pooled findings using random-effects models among patients with TBI indicated that intravenous administration of cerebrolysin significantly increased Glasgow Outcome Scale score (SMD $=0.30 ; 95 \%$ CI: 0.18 to $0.42 ; P<0.001 ; I^{2}: 87.8 \%$ ) and decreased modified Rankin Scale score (SMD $=-0.29 ; 95 \% \mathrm{CI}:-0.42$ to $0.16 ; P=0.05 ; P: 89.6 \%$ ).

Limitations: The results are mainly based on cohort studies and there is a lack of clinical trials in the literature. There is also heterogeneity among the studies regarding the dosage and duration of administration and the measurement of functional outcome.

Conclusion: The results of the current study revealed that intravenous administration of cerebrolysin is associated with improved functional outcome in patients with TBI measured by the Glasgow Outcome Scale and the modified Rankin Scale scores.

Keywords: traumatic brain injury, TBI, cerebrolysin, functional outcome, Glasgow Coma Scale, GOS, modified Rankin Scale, mRS

\section{Introduction}

Traumatic brain injury (TBI) is among the most common public health problems in both developed and developing countries being associated with high mortality and morbidity and heavy disease burden in all age groups. ${ }^{1,2}$ According to Center for Disease Control, TBI has been responsible for $\sim 2.5$ million emergency department 
visits, $\sim 282,000$ TBI-related hospitalizations, and $\sim 56,000$ TBI-related deaths. ${ }^{3}$ The incidence rate is as high as $1.9 \%$ in developed countries while it is even higher in developing countries with a TBI-associated mortality rate of 37.7-48.4 per 100,000 populations. ${ }^{46}$ The direct and indirect economic burden of the TBI is also a major and growing problem for the policy makers and stockholders with an estimated 4.4 trillion USD in gross domestic product losses during 2015-2030 in developing countries. ${ }^{7}$ The current treatment and management of TBI mainly focus on prevention of secondary brain injuries defined as ischemia, infarction, infection, and hydrocephalus along with rehabilitation, cognitive, and physical therapies. ${ }^{8} 9$ Several surgical and medical interventions have been introduced and clinically experienced in patients with TBI with various results; however, none of them have achieved favorable functional outcome and recovery. ${ }^{10-14}$ The research in the field is ongoing with introductions and establishment of new methods and techniques to improve the functional outcome of patients with TBI.

Cerebrolysin is a low molecular neuropeptide with neuroregenerative properties, which is prepared from the extract of the porcine brain tissue with specific laboratory and manufacturing considerations. In vitro and in vivo studies have demonstrated several beneficial effects of cerebrolysin, including decreased excitotoxicity, inhibiting free radical formation, microglial activation/neuroinflammation, and calpain activation/apoptosis. ${ }^{15}$ The other interesting effects of the cerebrolysin on neural tissue include inducing neural sprouting in tissue culture, neurotropic effects, and increased neural survival in ischemic conditions. ${ }^{16,17}$ The promising effects of cerebrolysin on regeneration of the neural tissue along with improved clinical and functional outcome of patients with ischemic strokes and neurodegenerative disorders, ${ }^{10,18}$ provided the hypothesis for its application in TBI. During the previous decade, several studies, mostly experimental, tried to investigate the effects of cerebrolysin on functional and clinical outcome of different grades of TBI. ${ }^{19-22}$ Despite the evidence provided from the animal studies were promising in models of TBI, the clinical studies provided controversial results. ${ }^{23-27}$ In addition, there is a scarce of high-quality clinical trials and cohort studies, which makes it hard to provide a level of evidence for application of cerebrolysin in TBI. The aim of the current systematic review and meta-analysis is to review all the available clinical data on cerebrolysin effects on functional outcome of patients with TBI and also analyze the results of cohort studies to reach a clear conclusion and evidence level. The participants are those with TBI, intervention is the administration of intravenous cerebrolysin, comparison is placebo, outcome is the functional outcome, and the study design is the clinical trial and cohort studies on the subject (patients, intervention, comparison, outcome, study design).

\section{Materials and methods}

Our study was performed and reported based on the guidelines outlined in the PRISMA.

\section{Search strategy and study selection}

To identify prospective studies investigating the impacts of cerebrolysin use on functional outcomes, two authors (F-GH and RT) independently conducted the searches in online databases, including Medline, Scopus, EMBASE, Google Scholar, Web of Science databases, and Cochrane Library from their inception to October 3, 2018. The following key terms were used for database searches; patients ("traumatic brain injury [TBI]" or "brain injury" or "head injury" or "mild TBI" or "moderate TBI" or "severe TBI" or "head trauma" or "brain trauma"), intervention ("cerebrolysin" and "intake" or "use" or "administration" or "application"), and functional outcomes ("Glasgow Outcome Scale [GOS]" or "modified Rankin Scale [mRS]" or "Glasgow Outcome Scale extended [GOSE]"). Reference lists of included articles and previous reviews were manually checked to obtain any further relevant studies not catching in electronic searches.

\section{Inclusion and exclusion criteria}

Articles were included in our meta-analysis that met the following inclusion criteria: studies published in a peerreviewed journal, original prospective articles and conducted on humans, investigated the effects of cerebrolysin use on functional outcomes among patients who had any forms of TBI. TBI was recently defined as: "An alteration in brain function, or other evidence of brain pathology, caused by an external force". ${ }^{28}$ The grading was done according to the on admission Glasgow Coma Scale (GCS) score: mild (14 and 15), moderate (9-13), and severe (3-8). Studies reported sufficient data to calculate the mean changes of scales, including GOS and mRS with related SDs between treatment and control groups. All articles including animal or in vitro studies, protocols without findings, congress abstracts without full texts, and articles published in a language other than English were excluded from the current meta-analysis.

\section{Data extraction and statistical analysis}

Two independent authors (F-GH and RT) extracted descriptive and numerical data from every included prospective 
article using a standard abstraction form of excel sheet. The extracted data for this meta-analysis consisted of the first authors' name, year of publication, and sample sizes in treatment and control groups, locations where study was conducted, the characteristics of patients, study design, dosage of cerebrolysin, duration of cerebrolysin, type of underlining disease, and functional outcomes of interest, including GOS or/and mRS measures means and SDs from treatment and control groups.

\section{Flowchart of study}

The flowchart of current systematic review identification and selection process has been presented in Figure 1. Overall, our initial search for prospective articles yielded a total of 29 reports. After step-by-step screening and reviewing, five prospective articles ultimately met our systematic review and meta-analysis inclusion criteria. ${ }^{23,29-32}$ Five included articles were consisted 5,685 patients, which were assigned to 765 participants in case group and 4,920 participants in control group. Of these articles, four articles used cohort design ${ }^{23,29,30,32}$ and one used randomized clinical trial design. ${ }^{31}$ Dosage of cerebrolysin use ranged from 10 to $30 \mathrm{~mL} /$ day. The included prospective studies were published between 2005 and 2017. Duration of cerebrolysin use varied from 10 days to 12 months. The Muresanu et $\mathrm{al}^{29}$ study reported the effects of cerebrolysin use on functional outcomes in varied conditions for duration, dosage, and underlying disease among participants; therefore, we included that article as several different studies. The detailed characteristics of included prospective articles are presented in Table 1.

\section{Statistical analysis}

Following extract, the required data, the mean differences with SD of functional outcomes between intervention and control groups were used to pool data. The standardized mean differences (SMDs) with corresponding 95\% CI, while adjusting by the method of Hedges g, were estimated as effect sizes using the random-effects model with

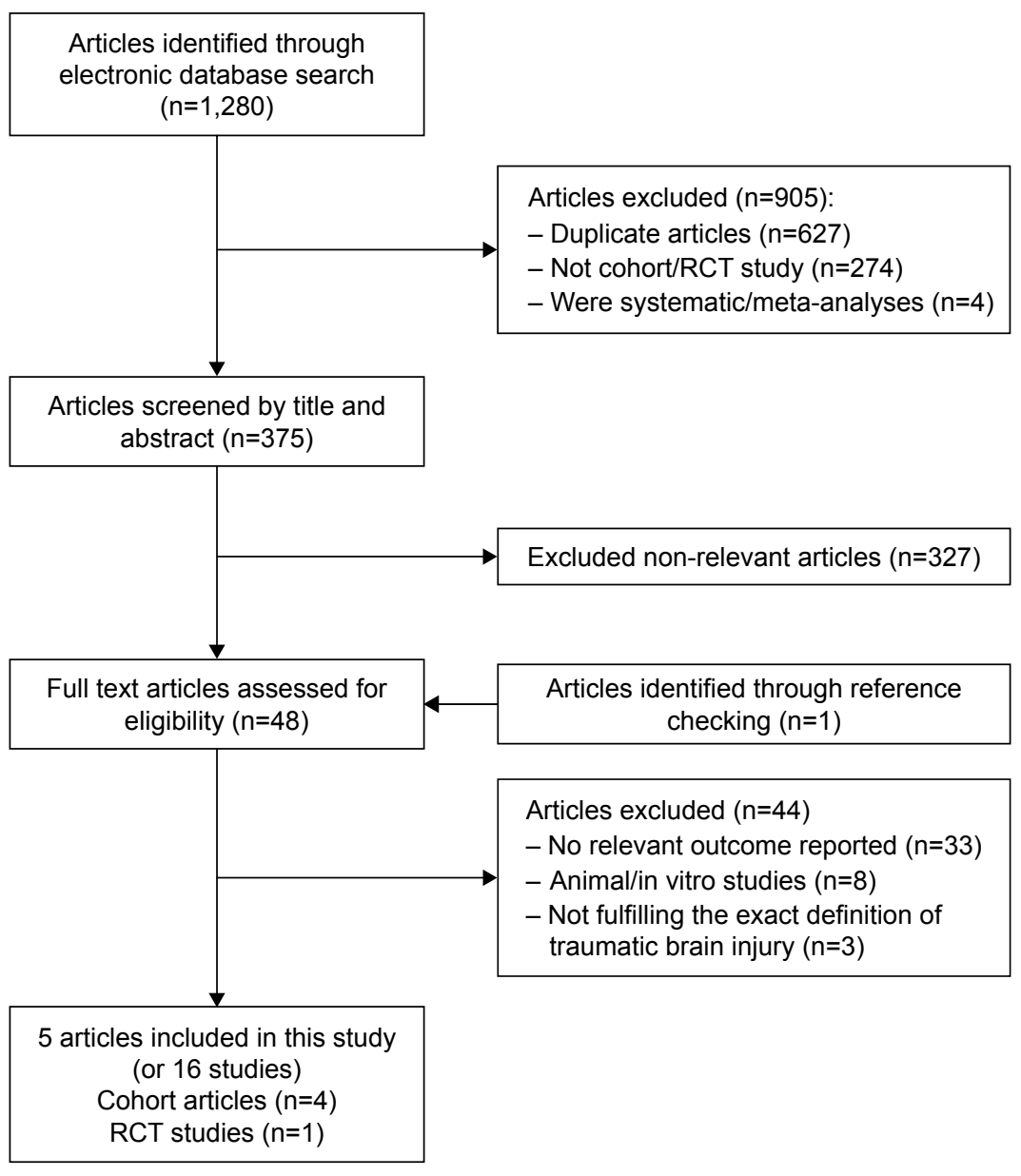

Figure I The flow diagram of the study identification and selection process. Abbreviation: RCT, randomized controlled trial. 


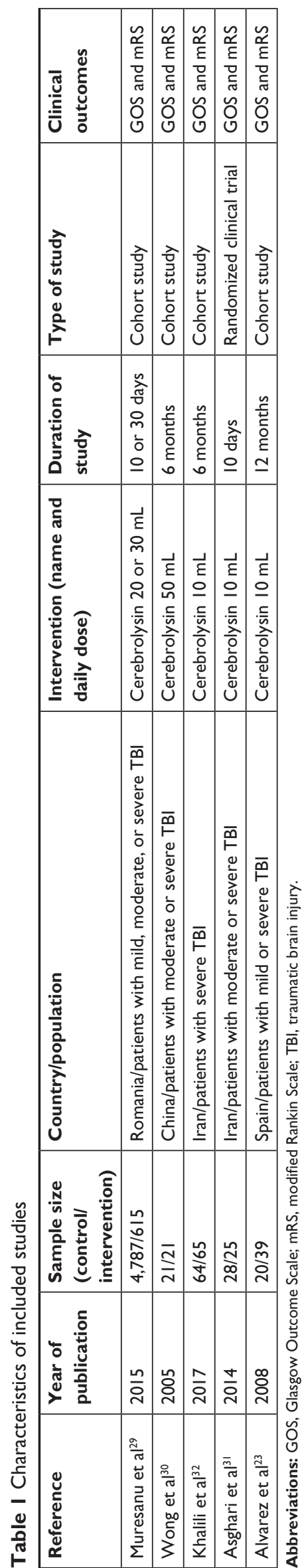

Dersimonian-Laird method according to the likely presence of significant heterogeneity across included primary studies. $I^{2}$ and Cochran's $Q$-statistics were used to assess heterogeneity. Sensitivity analyses were performed to estimate the impact of one-by-one study on reliability of the total combined SMDs for each of functional outcomes. Furthermore, subgroup analyses were done to assess the source of heterogeneity based on the possible moderator variables, including the following: type of disease (patients with mild TBI vs those with moderate to severe TBI), dosage of cerebrolysin ( $\leq 20 \mathrm{vs}>20 \mathrm{~mL} /$ day), and duration of study ( $\leq 10$ vs $10-30$ vs $>30$ days). Egger's and Begg's tests were applied to examine any potential publication bias in current meta-analysis. All related statistical analyses were calculated with STATA software version 12.0 (Stata Corp., College Station, TX, USA).

\section{Results}

\section{The association between cerebrolysin use} and functional outcomes

Forest plots demonstrating the impacts of cerebrolysin use on functional outcomes are indicated in Figure 2. Our results showed that cerebrolysin use in patients with TBI significantly increased GOS (SMD $=0.30 ; 95 \%$ CI: 0.18 to 0.42 ; $P<0.001 ; I^{2}: 87.8 \%$ ), and decreased $\mathrm{mRS}$ (SMD $=-0.29$; 95\% CI: -0.42 to $0.16 ; P=0.05 ; I^{2}: 89.6 \%$ ) (Table 2).

\section{Sensitivity/subgroup analyses}

Sensitivity analyses did not significantly change the overall combined SMDs on functional outcomes. So, the lower and higher pooled SMDs for GOS in the sensitivity analysis were 0.27 (95\% CI: 0.15 to 0.39 ) after removing the study by Muresanu et $\mathrm{al}^{29}$ and 0.33 (95\% CI: 0.21 to 0.44 ) after removing the study by Asghari et al. ${ }^{31}$ For mRS, the lower pooled SMD in the sensitivity analysis was -0.34 ( $95 \%$ CI: -0.46 to 0.22 ) after removing the study by Asghari et $\mathrm{al}^{31}$ and the higher was -0.26 ( $95 \% \mathrm{CI}:-0.39$ to 0.13 ) after removing the study by Khalili et al..$^{32}$ The findings of subgroup analyses indicated that heterogeneity between included studies was not significant both before and after subgroup analysis. However, the detailed results of the subgroup analyses by type of the disease, dosage of the cerebrolysin, and duration of the study are summarized in Table 3.

\section{Publication bias}

Egger's and Begg's statistics indicated no significant evidence of potential publication bias for meta-analysis estimating the impact of cerebrolysin use on functional outcomes, 


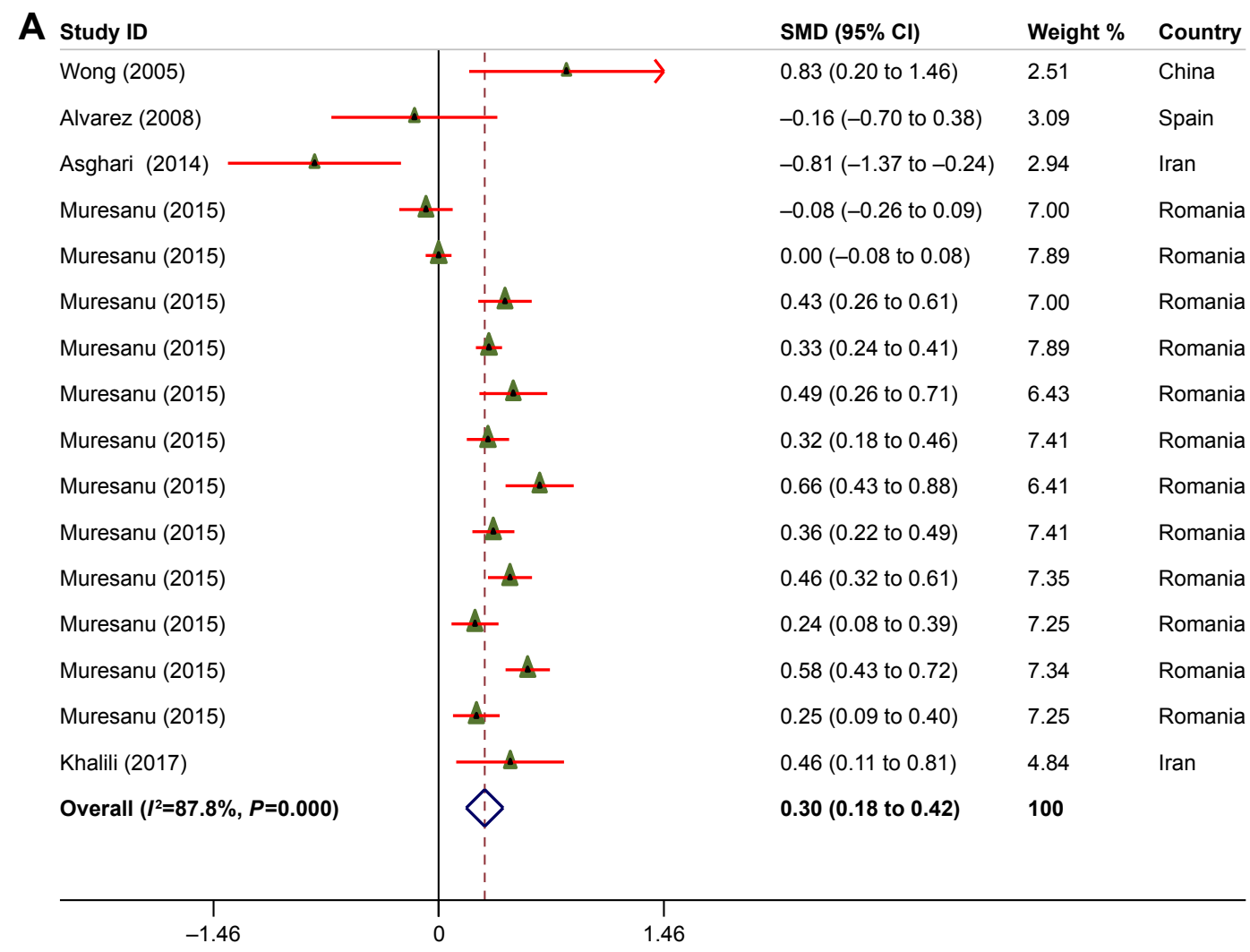

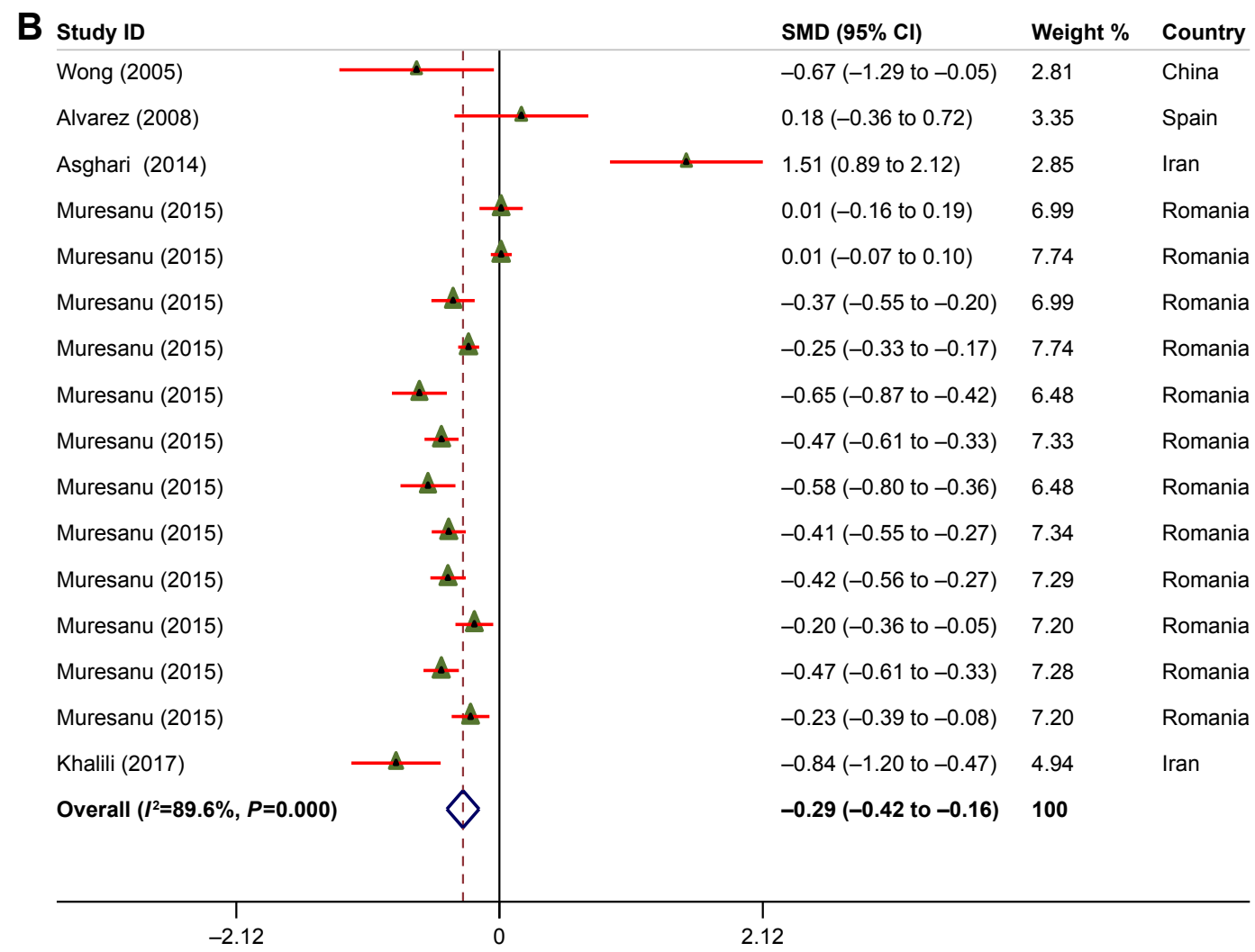

Figure 2 Meta-analysis on clinical outcomes (SMD estimates [95\% Cl]) for GOS (A) and mRS (B) scores for patients treated with cerebrolysin and control groups. Note: Weights are from random-effects analysis.

Abbreviations: GOS, Glasgow Outcome Scale; mRS, modified Rankin Scale; SMD, standardized mean difference. 
Table 2 The overall pooled SMD for cerebrolysin treatment across all included studies

\begin{tabular}{|c|c|c|c|c|c|c|c|}
\hline \multirow{2}{*}{$\begin{array}{l}\text { Clinical } \\
\text { outcomes }\end{array}$} & \multirow{2}{*}{$\begin{array}{l}\text { Number of } \\
\text { studies }\end{array}$} & \multirow{2}{*}{$\begin{array}{l}\text { Pooled } \\
\text { SMDs }\end{array}$} & \multirow[t]{2}{*}{$95 \% \mathrm{Cl}$} & \multirow[t]{2}{*}{$P$-value } & \multicolumn{3}{|c|}{ Heterogeneity } \\
\hline & & & & & $I^{2}(\%)$ & $Q$ & $P$-value heterogeneity \\
\hline GOS & 16 & 0.3 & 0.18 to 0.42 & $<0.001$ & 87.8 & 123.32 & $<0.001$ \\
\hline $\mathrm{mRS}$ & 16 & -0.29 & -0.42 to 0.16 & 0.05 & 89.6 & 143.67 & $<0.001$ \\
\hline
\end{tabular}

Abbreviations: GOS, Glasgow Outcome Scale; mRS, modified Rankin Scale; SMD, standardized mean difference.

including GOS (Begg's test $P=0.97$ and Egger's test $P=0.73$ ) and mRS (Begg's test $P=0.50$, Egger's test $P=0.32$ ).

\section{Discussion}

We ran this systematic review and meta-analysis to assess the efficacy of the cerebrolysin treatment on functional outcome of patients with TBI. The results of the current study demonstrate that intravenous administration of cerebrolysin after TBI is associated with improved functional recovery in patients with different grades of the TBI (mild to severe).
The functional outcome in the included studies was GOS and $\mathrm{mRS}$ and other indices, such as cognitive functions and memory could not be included in the pooled analysis due to lack of homogeneity and availability. The study demonstrates large heterogeneity in the performed studies and reported outcomes. Also, lack of standard clinical trials decreases the level of evidence for this therapy in patients with TBI. The efficacy and safety of cerebrolysin for management of TBI has been tested in several animal studies with favorable results. ${ }^{19-22}$ However, the clinical studies are scarce on

Table 3 The association between cerebrolysin use and clinical outcomes in patients with traumatic brain injury based on subgroup analysis

\begin{tabular}{|c|c|c|c|c|c|}
\hline Variables & $K^{a}$ & $I^{2}(\%)$ & Q test & SMD (95\% Cl) & $P$-value \\
\hline \multicolumn{6}{|l|}{ GOS } \\
\hline Total & 16 & 87.8 & 123.32 & 0.30 (0.18 to 0.42$)$ & $<0.001$ \\
\hline \multicolumn{6}{|l|}{ Type of disease } \\
\hline Mild TBI & 4 & 93.4 & 45.76 & $0.17(-0.06$ to 0.40$)$ & 0.14 \\
\hline Moderate to severe TBI & 12 & 76.0 & 45.79 & $0.36(0.24$ to 0.49$)$ & $<0.001$ \\
\hline \multicolumn{6}{|c|}{ Dosage of cerebrolysin (mL/day) } \\
\hline$>20$ & 7 & 86.1 & 43.32 & $0.45(0.25$ to 0.65$)$ & $<0.001$ \\
\hline$\leq 20$ & 9 & 85.1 & 54.96 & 0.20 (0.06 to 0.34$)$ & $<0.01$ \\
\hline \multicolumn{6}{|l|}{ Duration of study (days) } \\
\hline$\leq 10$ & 7 & 83.2 & 35.72 & $0.36(0.2 \mathrm{I}$ to $0.5 \mathrm{I})$ & 0.01 \\
\hline $10-30$ & 6 & 90.6 & 53.14 & $0.23(0.04$ to 0.42$)$ & $<0.001$ \\
\hline$>30$ & 3 & 67.6 & 6.18 & $0.37(-0.13$ to 0.87$)$ & 0.14 \\
\hline \multicolumn{6}{|l|}{ mRS } \\
\hline Total & 16 & 89.6 & 143.67 & $-0.29(-0.42$ to -0.16$)$ & 0.05 \\
\hline \multicolumn{6}{|l|}{ Type of disease } \\
\hline Mild TBI & 4 & 89.7 & 29.16 & $-0.15(-0.33$ to 0.04$)$ & 0.11 \\
\hline Moderate to severe TBI & 12 & 83.7 & 67.52 & $-0.36(-0.5 \mathrm{I}$ to $-0.2 \mathrm{I})$ & $<0.001$ \\
\hline \multicolumn{6}{|c|}{ Dosage of cerebrolysin (mL/day) } \\
\hline$>20$ & 7 & 80.4 & 30.60 & $-0.42(-0.59$ to -0.25$)$ & $<0.001$ \\
\hline$\leq 20$ & 9 & 89.6 & 92.99 & $-0.29(-0.42$ to -0.16$)$ & 0.03 \\
\hline \multicolumn{6}{|l|}{ Duration of study (days) } \\
\hline$\leq 10$ & 7 & 87.8 & 49.05 & $-0.28(-0.45$ to -0.05$)$ & $<0.01$ \\
\hline $0-30$ & 6 & 92.8 & 69.22 & $-0.28(-0.49$ to -0.06$)$ & 0.01 \\
\hline$>30$ & 3 & 97.2 & 9.47 & $-0.46(-1.09$ to 0.17$)$ & 0.15 \\
\hline
\end{tabular}

Note: ${ }^{\mathrm{K}} \mathrm{K}$ denotes number of SMD included.

Abbreviations: GOS, Glasgow Outcome Scale; mRS, modified Rankin Scale; SMD, standardized mean differences; TBI, traumatic brain injury. 
the subject and the literature only includes limited number of standard studies addressing the effectiveness of the cerebrolysin on functional recovery after various grades of TBI. ${ }^{23-25,29,30,32}$ The studies are mostly prospective or retrospective cohorts and only limited randomized clinical trials are available. ${ }^{27,31}$ Currently, a large multicenter, placebocontrolled, randomized clinical trial is underway to address the subject but the results are not available yet. ${ }^{33}$

The heterogeneity in the clinical studies could be classified in several fields. First, the inclusion criteria for the patients with TBI vary between these studies. While some authors have included only patients with severe TBI, ${ }^{32}$ other included moderate and severe TBI patients ${ }^{23,29-31}$ and Chen et $\mathrm{al}^{27}$ studied only mild TBI patients. These heterogeneous inclusion criteria led to limited power of analysis because the management and outcome of patients depend on the severity of TBI. The baseline GCS has been demonstrated to be the most important predictor of functional outcome in patients with TBI. ${ }^{34}$ Thus, cerebrolysin could have various effects on the outcome of patients with different grades of TBI. The other factor contributing to heterogeneity of the studies is the dosage and duration of the treatment. Some studies administered the cerebrolysin in $10 \mathrm{~mL} /$ day dosage ${ }^{23,31,32}$ while other used the $20-30 \mathrm{~mL} /$ day dosages. ${ }^{27,29}$ The highest dosage ( $50 \mathrm{~mL} /$ day) was given by Wong et al. ${ }^{30}$ The duration also varied between 5 and 30 days. As cerebrolysin is a newly investigated medication, the appropriate dosage and duration should be elucidated according to the pharmacokinetics and the clinical studies. ${ }^{15,25}$ The current treatment regimens are mainly based on the regimens that are given to the patients with ischemic stroke, ${ }^{16,18}$ Alzheimer's, ${ }^{35}$ and Parkinson's disease. ${ }^{36}$ König et al ${ }^{25}$ also reported the beneficial effects of cerebrolysin given at the dosage of $50 \mathrm{~mL} /$ day for 21 days in patients with various grades of TBI measured by serial GCS and cognitive tests. Thus, the standard treatment protocol for TBI should be established based on the ongoing research. The Cerebrolysin Asian Pacific Trial in Acute Brain Injury and Neurorecovery, which is underway, is using a $50 \mathrm{mg}$ /day dosage over a 10 -day period. ${ }^{33}$ And finally, the outcome measures of functional recovery are heterogeneous between the studies. Most of the studies report the GOS, GOSE, and $\mathrm{mRS}^{24,29-32}$ while Chen et $\mathrm{al}^{27}$ reported Mini-Mental State Examination (MMSE), and Cognitive Abilities Screening Instrument (CASI). This is due the fact that patients with mild TBI were included and GOS and $\mathrm{mRS}$ have limited value in evaluating the functional recovery in these patients. Thus, cognitive measurement outcomes, such as MMSE and CASI should be included. In addition, some other studies used the EEG findings to determine the effects of cerebrolysin on outcome in patients with mild TBI. ${ }^{23,24}$ These variations reduced the number of studies that could be included in the meta-analysis, although there was heterogeneity between the studies. The findings of subgroup analyses indicated that heterogeneity between included studies had no significant effects on the outcome measures before and after subgroup analysis. Furthermore, all the studies, which were not included, demonstrated that cerebrolysin therapy is associated with improved functional, cognitive, and electrical outcomes in patients with mild, moderate, and severe TBI.

The neuroprotective and neuroregenerative properties of the cerebrolysin have increased its use in different neurologic and neurosurgical conditions. Recently, Hassanein et al ${ }^{37}$ demonstrated that cerebrolysin administration dramatically improved the communication defects of the neonates with severe perinatal brain insults. These results along with those previously reported on effectiveness of cerebrolysin on regeneration of injured nerves ${ }^{38}$ suggest that the proposed treatment could be used along with stem cell therapies to induce neuroregeneration. The horizons of neural therapy with stem cell and regenerative medicine could be established by use of cerebrolysin. Most recently, Zhang et $\mathrm{al}^{20}$ reported the results of a prospective, randomized, placebo-controlled study of cerebrolysin dose-dependent effects in animal model of mild TBI. They demonstrated that administration of cerebrolysin at doses of $0.8-7.5 \mathrm{~mL} / \mathrm{kg}$, administered 4 hours after mild TBI and then once daily for a total of 10 consecutive days, improved functional outcomes 3 months after injury. ${ }^{20}$ In a similar study, Sharma et al ${ }^{19}$ investigated the beneficial effects of cerebrolysin therapy on pathophysiological aspects of TBI. They demonstrated that administration of cerebrolysin 5 minutes or 1 hour after TBI reduced blood-brain barrier and blood-cerebrospinal fluid barriers permeability changes, attenuated brain pathology and brain edema, and mitigated functional deficits. However, administration of cerebrolysin 2 hours after the injury had no beneficial effects. These findings suggest timely intervention with cerebrolysin to achieve appropriate functional outcome. ${ }^{19}$ Taking all these together, it could be concluded that timely and dose-dependent effects of cerebrolysin should be considered in designing trials in TBI. The results of the current meta-analysis also confirm the efficacy of the cerebrolysin therapy in improving the functional outcome of patients with various grades of TBI.

\section{Conclusion}

Intravenous cerebrolysin administration has been associated with improved functional recovery after TBI as measured by 
$\mathrm{mRS}$ and GOS. There is a significant heterogeneity in dosage and duration of therapy as well as interval between the injury and treatment application, which limits the effect analysis. In addition, the various grades of TBI (mild, moderate, and severe) seem to benefit from cerebrolysin therapy after the injury with regard to the functional outcome indices. The lack of standard and high quality randomized clinical trials limits the clinical application and final conclusion on the efficacy of cerebrolysin in patients with TBI and the level of evidence remains low in results. Based on the results of the current study, intravenous could be recommended for treatment of patients with different grades of TBI with level II of evidence. More randomized clinical trials are recommended to elucidate the issue.

\section{Acknowledgment}

We would like to acknowledge the Diba Negar Research Institute for editorial assistance and improving the style and English in the manuscript.

\section{Disclosure}

The authors report no conflicts of interest in this work.

\section{References}

1. Thurman DJ. The epidemiology of traumatic brain injury in children and youths: a review of research since 1990. J Child Neurol. 2016; 31(1):20-27.

2. Cole TB. Global road safety crisis remedy sought: 1.2 million killed, 50 million injured annually. JAMA. 2004;291(21):2531-2532.

3. Taylor CA, Bell JM, Breiding MJ, Xu L. Traumatic brain injury-related emergency department visits, hospitalizations, and deaths - United States, 2007 and 2013. MMWR Surveill Summ. 2017;66(9):1-16.

4. Heydari ST, Hoseinzadeh A, Ghaffarpasand F, et al. Epidemiological characteristics of fatal traffic accidents in Fars province, Iran: a community-based survey. Public Health. 2013;127(8):704-709.

5. Heydari ST, Hoseinzadeh A, Sarikhani Y, et al. Time analysis of fatal traffic accidents in Fars Province of Iran. Chin J Traumatol. 2013;16(2): 84-88.

6. Peymani P, Heydari ST, Hoseinzadeh A, et al. Epidemiological characteristics of fatal pedestrian accidents in Fars province of Iran: a community-based survey. Chin J Traumatol. 2012;15(5):279-283.

7. Rudolfson N, Dewan MC, Park KB, Shrime MG, Meara JG, Alkire BC. The economic consequences of neurosurgical disease in low- and middle-income countries. J Neurosurg. Epub May 18, 2018.

8. Carney N, Totten AM, O'Reilly C. Guidelines for the management of severe traumatic brain injury, fourth edition. Neurosurgery. 2017; 80(1):6-15.

9. Jia K, Tong X, Liang F. Effects of sequential nutritional support on nutritional status and expression of regulatory $\mathrm{T}$ lymphocyte in patients with early severe traumatic brain injury. Neuropsychiatr Dis Treat. 2018; 14:1561-1567.

10. El Sayed I, Zaki A, Fayed AM, Shehata GM, Abdelmonem S. A meta-analysis of the effect of different neuroprotective drugs in management of patients with traumatic brain injury. Neurosurg Rev. 2018;41(2):427-438.

11. Sun HT, Zheng M, Wang Y, Diao Y, Zhao W, Wei Z. Monitoring intracranial pressure utilizing a novel pattern of brain multiparameters in the treatment of severe traumatic brain injury. Neuropsychiatr Dis Treat. 2016;12:1517-1523.
12. Farzanegan GR, Derakhshan N, Khalili H, Ghaffarpasand F, Paydar S. Effects of atorvastatin on brain contusion volume and functional outcome of patients with moderate and severe traumatic brain injury; a randomized double-blind placebo-controlled clinical trial. J Clin Neurosci. 2017;44:143-147.

13. Khalili H, Derakhshan N, Niakan A, et al. Effects of oral glibenclamide on brain contusion volume and functional outcome of patients with moderate and severe traumatic brain injuries: a randomized double-blind placebo-controlled clinical trial. World Neurosurg. 2017;101:130-136.

14. Khalili H, Niakan A, Ghaffarpasand F, Kiani A, Behjat R. Outcome determinants of decompressive craniectomy in patients with traumatic brain injury; a single center experience from southern Iran. Bull Emerg Trauma. 2017;5(3):190-196.

15. Masliah E, Díez-Tejedor E. The pharmacology of neurotrophic treatment with Cerebrolysin: brain protection and repair to counteract pathologies of acute and chronic neurological disorders. Drugs Today. 2012;48(Suppl A):3-24.

16. Zhang L, Chopp M, Meier DH, et al. Sonic hedgehog signaling pathway mediates cerebrolysin-improved neurological function after stroke. Stroke. 2013;44(7):1965-1972.

17. Ruozi B, Belletti D, Sharma HS, et al. PLGA nanoparticles loaded cerebrolysin: studies on their preparation and investigation of the effect of storage and serum stability with reference to traumatic brain injury. Mol Neurobiol. 2015;52(2):899-912.

18. Amiri-Nikpour MR, Nazarbaghi S, Ahmadi-Salmasi B, Mokari T, Tahamtan U, Rezaei Y. Cerebrolysin effects on neurological outcomes and cerebral blood flow in acute ischemic stroke. Neuropsychiatr Dis Treat. 2014;10:2299-2306.

19. Sharma HS, Zimmermann-Meinzingen S, Johanson CE. Cerebrolysin reduces blood-cerebrospinal fluid barrier permeability change, brain pathology, and functional deficits following traumatic brain injury in the rat. Ann N Y Acad Sci. 2010;1199:125-137.

20. Zhang Y, Chopp M, Gang Zhang Z. Prospective, randomized, blinded, and placebo-controlled study of cerebrolysin dose-response effects on long-term functional outcomes in a rat model of mild traumatic brain injury. J Neurosurg. Epub Jan 5, 2018.

21. Zhang Y, Chopp M, Meng Y, et al. Improvement in functional recovery with administration of cerebrolysin after experimental closed head injury. J Neurosurg. 2013;118(6):1343-1355.

22. Zhang Y, Chopp M, Meng Y, et al. Cerebrolysin improves cognitive performance in rats after mild traumatic brain injury. $J$ Neurosurg. 2015;122(4):843-855.

23. Alvarez XA, Sampedro C, Figueroa J, et al. Reductions in qEEG slowing over 1 year and after treatment with Cerebrolysin in patients with moderate-severe traumatic brain injury. J Neural Transm. 2008; 115(5):683-692.

24. Alvarez XA, Sampedro C, Pérez P, et al. Positive effects of cerebrolysin on electroencephalogram slowing, cognition and clinical outcome in patients with postacute traumatic brain injury: an exploratory study. Int Clin Psychopharmacol. 2003;18(5):271-278.

25. König $P$, Waanders R, Witzmann A. Cerebrolysin in traumatic brain injury - a pilot study of a neurotrophic and neurogenic agent in the treatment of acute traumatic brain injury. J Neurol Neurochir Psychiatr. 2006;7:12-20.

26. Onose G, Mureşanu DF, Ciurea AV, et al. Neuroprotective and consequent neurorehabilitative clinical outcomes, in patients treated with the pleiotropic drug cerebrolysin. J Med Life. 2009;2(4):350-360.

27. Chen CC, Wei ST, Tsaia SC, Chen XX, Cho DY. Cerebrolysin enhances cognitive recovery of mild traumatic brain injury patients: doubleblind, placebo-controlled, randomized study. Br J Neurosurg. 2013; 27(6):803-807.

28. Menon DK, Schwab K, Wright DW, Maas AI. Demographics and clinical assessment working group of the International and interagency initiative toward common data elements for research on traumatic brain injury and psychological health. Position statement: definition of traumatic brain injury. Arch Phys Med Rehabil. 2010;91(11):1637-1640. 
29. Muresanu DF, Ciurea AV, Gorgan RM, et al. A retrospective, multicenter cohort study evaluating the severity-related effects of cerebrolysin treatment on clinical outcomes in traumatic brain injury. CNS Neurol Disord Drug Targets. 2015;14(5):587-599.

30. Wong GK, Zhu XL, Poon WS. Beneficial effect of cerebrolysin on moderate and severe head injury patients: result of a cohort study. Acta Neurochir Suppl. 2005;95:59-60.

31. Asghari M, Meshkini A, Salehpoor F, et al. Investigation of the effect of cerebrolysin on patients with head trauma and diffuse axonal injury. Int J Curr Res Acad Rev. 2014;2:1-8.

32. Khalili H, Niakan A, Ghaffarpasand F. Effects of cerebrolysin on functional recovery in patients with severe disability after traumatic brain injury: a historical cohort study. Clin Neurol Neurosurg. 2017; 152:34-38

33. Poon W, Vos P, Muresanu D, et al. Cerebrolysin Asian Pacific trial in acute brain injury and neurorecovery: design and methods. $\mathrm{J} \mathrm{Neu}$ rotrauma. 2015;32(8):571-580.
34. Mcnett M, Amato S, Gianakis A, et al. The FOUR score and GCS as predictors of outcome after traumatic brain injury. Neurocrit Care. 2014; 21(1):52-57.

35. Gauthier S, Proaño JV, Jia J, Froelich L, Vester JC, Doppler E. Cerebrolysin in mild-to-moderate Alzheimer's disease: a meta-analysis of randomized controlled clinical trials. Dement Geriatr Cogn Disord. 2015; 39(5-6):332-347.

36. Noor NA, Mohammed HS, Mourad IM, Khadrawy YA, Aboul Ezz HS. A promising therapeutic potential of cerebrolysin in 6-OHDA rat model of Parkinson's disease. Life Sci. 2016;155:174-179.

37. Hassanein SM, Deifalla SM, El-Houssinie M, Mokbel SA. Safety and efficacy of cerebrolysin in infants with communication defects due to severe perinatal brain insult: a randomized controlled clinical trial. J Clin Neurol. 2016;12(1):79-84.

38. Stepanichev M, Onufriev M, Aniol V, et al. Effects of cerebrolysin on nerve growth factor system in the aging rat brain. Restor Neurol Neurosci. 2017;35(6):571-581.
Neuropsychiatric Disease and Treatment

\section{Publish your work in this journal}

Neuropsychiatric Disease and Treatment is an international, peerreviewed journal of clinical therapeutics and pharmacology focusing on concise rapid reporting of clinical or pre-clinical studies on a range of neuropsychiatric and neurological disorders. This journal is indexed on PubMed Central, the 'PsycINFO' database and CAS,

\section{Dovepress}

and is the official journal of The International Neuropsychiatric Association (INA). The manuscript management system is completely online and includes a very quick and fair peer-review system, which is all easy to use. Visit http://www.dovepress.com/testimonials.php to read real quotes from published authors.

Submit your manuscript here: http://www.dovepress.com/neuropsychiatric-disease-and-treatment-journal 